\title{
SMALL NEIGHBORHOODS OF THE IDENTITY OF A REAL NILPOTENT GROUP1
}

\author{
L. P. POLEK
}

\begin{abstract}
It is shown that if $G$ is a real nilpotent group of type $\mathrm{D}$, then for every neighborhood $U$ of the identity in $G$ there is a discrete cocompact subgroup $\Gamma_{U}$ of $G$ such that for every $\varphi \in$ Aut $(G), \varphi \Gamma_{U}$ and $U$ have more elements in common than just the identity.

This result is exactly the opposite of what is true when $G$ is a semisimple Lie group.
\end{abstract}

1. Introduction. A Lie group $G$ shall mean a connected Lie group. $\hat{G}$ will denote its Lie algebra and $\operatorname{Aut}(G)$ its group of continuous automorphisms with the $C-0$ topology. Call $G$ a real nilpotent group if it is a simply connected, nilpotent, real Lie group.

We shall consider discrete cocompact subgroups $\Gamma$ of $G$ so that $G / \Gamma$ has a finite measure invariant under the action of $G$. Let $\Phi(G)$ denote the totality of such $\Gamma$ 's in $G$. Let $\mu$ be a Haar measure on $G$. A Borel set $P$ in $G$ is a $\Gamma$-packing if $P \cap P \gamma=\varnothing$ for every $\gamma \in \Gamma, \gamma \neq e . v(\Gamma)=\mu(G / \Gamma)$ shall be called the volume of $\Gamma$.

Suppose $E$ is a $\mu$-measurable subset of $G$ and $\alpha \in \operatorname{Aut}(G)$. Then $\mu(\alpha(E))=\Delta(\alpha) \mu(E)$, where $\Delta: \operatorname{Aut}(G) \rightarrow R_{>0}$ is a homomorphism into the multiplicative group of the positive reals. If $G$ is a real nilpotent group, then $\Delta(\alpha)=\mid$ det $\alpha \mid$, since we can identify $G$ with its Lie algebra $\hat{G}$, and $\operatorname{Aut}(G)$ with $\operatorname{Aut}(\hat{G})$. Call $G$ totally unimodular if the image of $\Delta$ is $\{1\}$.

Definition 1. Let $\hat{G}$ be a nilpotent Lie algebra over $k$, charac $k=0$. $\hat{G}$ will be called a Lie algebra of type $\mathrm{D}$ if every derivation of $\hat{G}$ is nilpotent. Call the real nilpotent group $\exp (\hat{G})=G$ a group of type $\mathrm{D}$. [See [2] for an example.]

The following is known.

(1.1) If $\hat{G}$ is a real Lie algebra of type $\mathrm{D}$, then $G=\exp (\hat{G})$ is totally unimodular.

Received by the editors March 15, 1973.

AMS (MOS) subject classifications (1970). Primary 22E25, $22 \mathrm{E} 40$.

1 These results were contained in the author's doctoral thesis written under the direction of Professor H. C. Wang at Cornell University.

(c) American Mathematical Society 1974 
DEFINITION 2. A nilpotent Lie group $G$ has an expanding automorphism if there exists an automorphism $\alpha$ of $G$ such that with respect to some basis, $\alpha$ has the form

$$
\alpha=\operatorname{diag}\left(a_{1}, a_{2}, \cdots, a_{n}\right) \text {, with each }\left|a_{i}\right|>1 .
$$

Definition 3. A Lie group $G$ has a $K M N$ if there is a nbhd $U$ of the identity $e$ of $G$ such that for each $\Gamma$ in $\Phi(G)$ there exists a $\varphi \in \operatorname{Aut}(G)$ such that $\varphi \Gamma \cap U=\{e\}$. Call $U$ a KMN of $G$.

In [3] it was shown that a semisimple Lie group without compact factors has a $\mathrm{KMN}$ and the automorphisms $\varphi$ are all inner automorphisms. We shall show that for some real nilpotent groups the opposite phenomenon is true.

2. Main theorem. In what follows $G$ will denote a real nilpotent group with a discrete cocompact subgroup $\mathrm{I}$.

Suppose $X_{1}, X_{2}, \cdots, X_{n}$ is a basis of $\hat{G}$. Call $O_{M}=\left\{\sum t_{i} X_{i} \mid 0 \leqq t_{i}<M\right\}$ the $M$-ball at 0 in $\hat{G}$ and $\exp O_{M}$ the $M$-ball at $e \in G$.

DEFINITION 4. A neighborhood $V$ of $e$ in $G$ is confined if $V \subset \exp O_{M}$ for some $M>0$.

This property of nbhds of $e$ is clearly independent of the chosen basis.

(2.1) If $G$ has an expanding automorphism then every confined nhhd of $e$ is a $K M N$.

Proof. Let $V$ be a confined nbhd and $\Gamma^{\prime} \in \Phi(G)$. Choose $M$ so that $V \subset \exp O_{M}$. Let $\alpha=\operatorname{diag}\left(a_{1}, a_{2}, \cdots, a_{n}\right)$ be an expanding automorphism. Now pick $m \in Z^{+}$large enough so that $\alpha^{m}=\operatorname{diag}\left(a_{1}^{m}, a_{2}^{m}, \cdots, a_{n}^{m}\right)$ maps $\Gamma-\{e\}$ outside of $\exp O_{M}$. Then $\alpha^{m} \Gamma \cap V=\{e\}$.

As an immediate corollary we have

(2.2) If $\hat{G}$ is quasi-cyclic, then every confined nbhd of e is a $K M N$ of $G$. (See [4] for the definition of quasi-cyclic.)

Our main theorem is

ThForfa 1. Suppose $G$ is of type D. For each nhhd $U$ of $e$ in $G$ there exists some $\Gamma_{\|}$in $\Phi(G)$ such that, for every $\psi \in \operatorname{Aut}(G), \gamma\left(\Gamma_{U}\right) \cap U \neq\{e\}$.

Proof. Suppose the theorem is false and $U$ is a $\mathrm{KMN}$ for $G$. Choose $V$, a nbhd of $c$, so that $V V \mathcal{L} \subset U$. Then $V$ is a $q\left(\Gamma^{l}\right)$-packing for each $\Gamma$ in $\Phi(G)$ and for some $q \in \operatorname{Aut}(G), q$, dependent on the chosen $\Gamma$. Take $\Gamma$ such that $I \cap V=\{e\}$. Now for every positive integer $n$ we can produce a $\Gamma_{n}$ in $D(G), \Gamma_{n} \supset \Gamma$, and such that the index of $\Gamma$ in $\Gamma_{n},\left[\Gamma_{n}: \Gamma\right]$, is greater than $n$ (for instance, by taking a canonical basis element in the 
center and shrinking it by an appropriate constant factor). Then $v(\mathrm{I})=$ $\left[\Gamma_{n}: \mathrm{l}\right] v\left(\Gamma_{n}\right)$, so that $v\left(\Gamma_{n}\right)<v\left(\Gamma^{\top}\right) / n$. Take $N$ large enough so that $v\left(\Gamma^{\prime}\right) / n<$ $\mu(V)$. Then $v\left(\Gamma_{N}\right)<\mu(V)$. Since $U$ is a $\mathrm{KMN}$, there is a $\varphi \in \operatorname{Aut}(G)$ with $\varphi\left(\Gamma_{N}\right) \cap U=\{e\}$. Since $G$ is type $\mathrm{D}$, by $(1.1)$ we have

$$
v\left(\Gamma_{N}\right)=v\left(\psi\left(\Gamma_{N}\right)\right)<\mu(V) .
$$

But $V$ is a $\varphi\left(\Gamma_{N}\right)$-packing, so this last relationship is a contradiction.

3. A stronger theorem. Let $\Phi_{c}(G)$ denote the subset of $\Phi(G)$ consisting of those $\Gamma$ 's for which $v\left(\Gamma^{\prime}\right) \geqq c>0$.

Definition 5. $G$ has a weak $\mathrm{KMN}$ if given any $c>0$ there exists a nbhd $W$ of $e$ in $G$ such that for every $\Gamma$ in $\Phi_{c}(G)$ there exists $\varphi \in \operatorname{Aut}(G)$ with $\varphi(\Gamma) \cap W=\{e\}$.

A real nilpotent group may not even have a weak $\mathrm{KMN}$.

THEOREM 2. Suppose $G$ is real nilpotent and totally unimodular. Fix $c>0$. Then for every nbhd $U$ of $e$ in $G$, there exists $a \Gamma$ in $\Phi_{c}(G)$ with $\varphi(\Gamma) \cap U \neq\{e\}$ for every $\varphi \in \operatorname{Aut}(G)$.

Proof. Suppose the theorem is false, i.e., assume $G$ has a weak KMN. Then we can show

Given $c>0$, there exist $\Gamma_{1}, \Gamma_{2}, \cdots, \Gamma_{s}$ in $\Phi(G)$ such that

(*) for every $D$ in $\Phi(G)$ with $v(D) \leqq c$, there exists a $\varphi \in \operatorname{Aut}(G)$ such that $\phi(D) \cong \mathrm{l}_{i}$, for some $i=1, \cdots, s$.

Assume (*) for the moment. Using known results it can be shown that, for any given $c>0$, there are infinitely many nonisomorphic l"s in $\Phi(G)$ with $v\left(\Gamma^{\prime}\right) \leqq c$, a contradiction of $(*)$. So, it is enough to prove $(*)$, given that $G$ has a weak KMN.

Proof of (*). Suppose, in fact, that $(*)$ is false. Then we can find a sequence $\left\{\mathrm{I}_{i}\right\}$ such that $v\left(\mathrm{I}_{i-1}\right) \leqq v\left(\mathrm{I}_{i}\right)$ for all $i$, and $\lim v\left(\mathrm{I}_{i}\right)=b \leqq c$. Set $v\left(\mathrm{l}_{1}\right)=c_{1}$. Then there exists $U_{1}$ a nbhd of $e$ and $U_{1}$ is a $\mathrm{KMN}$ for $\bar{\Phi}_{c_{i}}(G)$. In particular, for each $\Gamma_{i}$ in our sequence there is a $\varphi_{i} \in \operatorname{Aut}(G)$ such that $\varphi_{i}\left(\mathrm{l}_{i}\right) \cap U_{1}=\{e\}$. Take the new sequence $\left\{\varphi_{i}\left(\mathrm{l}_{i}\right)\right\}$ which is uniformly discrete and $v\left(\varphi_{i}\left(\Gamma_{i}\right)\right) \leqq c$, for each $i$, since $G$ is totally unimodular. Now by a theorem of Chabauty [1] there exists a convergent subsequence. The proof now follows the same line of reasoning as $\mathrm{H}$. C. Wang's (8.1) Theorem, in [6].

\section{REFERENCES}

1. C. Chabauty, Limite d'ensembles et géométrie des numbres, Bull. Soc. Math. France 78 (1950), 143-151. MR 12, 479.

2. J. Dixmier and W. G. Lister, Derivations of nilpotent Lie algebras, Proc. Amer. Math. Soc. 8 (1957), 155-158. MR 18, 659. 
3. D. A. Každan and G. A. Margulis, A proof of Selberg's conjecture, Mat. Sb. 75 (117) (1968), 163-168= Math. USSR Sb. 4 (1968), 147-152. MR 36 \#6535.

4. G. Leger, Derivations of Lie algebras. III, Duke Math. J. 30 (1963), 637-645. MR 28 \#3064.

5. A. I. Mal'cev, On a class of homogeneous spaces, Izv. Akad. Nauk SSSR Ser. Mat. 13 (1949), 9-32; English transl., Amer. Math. Soc. Transl. (1) 9 (1962), 276-307. MR 10, 507.

6. H. C. Wang, Topics on totally discontinuous groups, Short Courses in Symmetric Spaces, Dekker, New York, 1972.

Department of Mathematics, Rutgers University, Camden, New Jersey 08102 